\title{
Fruit in The Prevention of Cancer
}

\author{
VV Zakrevskii* \\ North-western State Medical University named after I.I. Mechnikov, Russia
}

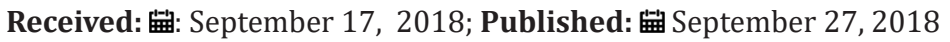

*Corresponding author: VV Zakrevskii, North-western State Medical University named after I.I. Mechnikov, Sankt-Petersburg, Russia

\begin{abstract}
Surveys of existing literature analyzed the effect of vegetables and fruits on the risk of developing various types of cancer justified the possibility of their use for the prevention of the risk of cancer. Apples and citrus have the most pronounced prophylactic properties along with dark grapes, blueberries, black currants, and blackberries, vegetables containing carotenoids (tomatoes, carrots, pumpkin, etc.), as well as onion, cruciferous and leafy vegetables. Dietary fiber is one of the most effective anti-cancer components in food composition. The anticancer effects of dietary fiber in dietary supplements has not been not provided, and sometimes resulted in the increase of risk of certain cancers. Also installed the anti-cancer effect for many of the phytonutrients included in the composition of different vegetables and fruits - antioxidants, carotenoids, folate, flavonoids, isothiocyanates, resveratrol, allicin and diallyl sulfide, but not for biologically active additives (dietary supplements) containing these compounds. The dangers of excessive consumption of such nutrients as selenium, alpha-tocopherol and omega-3 fatty acids, which, in particular, increased the risk of developing prostate cancer, carotenoids the risk lung cancer, and a comprehensive dietary Supplement with antioxidants the risk of bladder cancer were observed. Vegetables, preserved by salting and pickling, as well as fried, increase the risk of developing cancer mainly of the digestive system.
\end{abstract}

Keywords: Vegetables; Fruits; Berries; Prevention of Cancer

\section{Introduction}

Among the causes of death on the planet, oncological diseases are in second place. Their prevention, especially nutritional, has long attracted the attention of scientists. The English oncology epidemiologists, R Doll \& R Pito [4], proved in the early 1980s that the share of nutrition among all risk factors for carcinogenesis is $35 \%$. Their work has given impetus to further research in this direction. However, up to now, information on the effect of vegetables and fruits on the risk of emergence and progression of cancers of various organs is quite controversial - from complete denial of their usefulness in cancer prevention to treating them almost as a panacea. This study aims at analyzing data effect of vegetables and fruits on the risk of development of various types of oncology diseases and the rationale for their use in cancer prevention.

\section{Materials and Methods}

This paper uses a method of literary studies following contemporary national and foreign publications. More than 300 literary sources have been reviewed. Patent search extent is 37 years. However, it is over last 5 years that most of papers dealing with the subject have been published.

\section{Results and Discussion}

Vegetables and fruits represent food products that a very diverse in terms of their chemical composition and mechanisms of therapeutic and preventive action. The studies on nutrition and cancer, summarized in the WHO report "Diet, Nutrition and the Prevention of Chronic Diseases" [2] before 2002, a possible association was shown for fruits and vegetables to reduce the risk of cancer of the oral cavity, esophagus, stomach, colon and rectum, an alleged association - for dietary fiber, vitamins C, A, E and folic acid, carotenoids, selenium, flavonoids, isoflavones, and lignans. At the same time, it has been established that canned salt products (including fruits and vegetables) might contribute to the likely risk of stomach cancer. What has changed over the period from 2002 to 2017 ? Since 2002, many studies have been carried out to clarify the effect of nutritional risk factors and the prevention of cancer, and a large number of analytical reviews on the subject have been written [3-7].

Publications of this period have demonstrated that the influence of fruits and vegetables on the risk of development of different types of cancer is different, sometimes significantly, especially among residents of different regions of the globe. In addition, different fruits and vegetables determine the risk of cancer and mortality following no particular pattern. For example, X Wang et al. [8] note that as of 2013 , no data that high consumption of fruits and vegetables will reduce the risk of cancer were firmly established. In their discussion, the researchers point out that, apparently:

a) the importance of fruits and vegetables in reducing mortality due to cancer should be assessed in conjunction with 
other risk factors (obesity, physical activity, smoking, alcohol drinking);

b) it is necessary to analyze the effect of fruits and vegetables, including their individual groups, on mortality and reducing the risk of cancer of various organs, and not just general oncological morbidity.

At the same time, analytical review of SA Smith-Warner et al. [9] provide convincing data on reducing the risk of cancer of the oral cavity and pharynx, esophagus, lungs and stomach by means of vegetables and fruits, a possible reduction of the risk of cancer of larynx, pancreas, breast and bladder, as well as the anticipated prophylactic effect in cancer of endometrium, cervical cancer and thyroid cancer. In Poland [10], a statistically significant reduction in the risk of breast cancer with an increase in the consumption of raw vegetables and fresh fruits was also observed, while in Europe, comparison of dietary regimens of women from 10 countries has established reliable inverse relationship between total consumption of fruits and vegetables ( $p=0.01)$, as well as vegetables $(p<0.01)$ and the risk of development of breast cancer.

The most pronounced preventive properties are found in apples and citrus fruits, dark grape varieties, blueberries, black currants, raspberries (especially black raspberry), vegetables containing carotenoids (tomatoes, carrots, pumpkins, etc.), and onion, cruciferous and leafy vegetables. Thus, the possibility of using certain berries (black raspberry powder, black currant and blueberry extracts) is shown to prevent and treat colorectal cancer due to their ability to suppress inflammation, oxidative stress, proliferative processes, and angiogenesis [11-13]. One of the most effective anti-cancer components is dietary fibers, which contribute significantly to the reduction of the risk of breast cancer, colorectal cancer, pancreatic cancer, cancer of esophagus, stomach, as well as the prevention of relapse and increase in the life expectancy of breast cancer patients [14]. Dietary fibers of different products have an unequal protective effect on cancer morbidity, that is, to prevent various cancers food fibers of different foods are needed. Dietary fibers supplied with biologically active dietary supplements did not show anti-cancer effect; moreover, sometimes they increased the risk of certain oncological diseases.

A systematic review of 25 prospective cohort and case-control studies with a meta-analysis of the association of risk of colorectal cancer with the amount of dietary fiber consumed showed a $10 \%$ reduction in the risk of colorectal cancer per $10 \mathrm{~g}$ of dietary fiber per day [14]. Amongst the mechanisms of beneficial effect of dietary fibers, the authors name the increase in the volume of fecal matter resulting in dilution of fecal carcinogens in the lumen of the gut and reduction in the transit time of carcinogens, as well as increase in the production of short-chain fatty acids by probiotic microflora. It has been established that many phytonutrients that are part of various vegetables and fruits, i.e. antioxidants (vitamins C, E, selenium, etc.), carotenoids (lycopene, alpha and beta carotene, zeaxanthin, etc.), folates, flavonoids, isothiocyanates, resveratrol, allicin, and dialysulphide, but not the dietary supplements containing these compounds, have anti-cancer effect. Currently, a danger of excessive consumption of nutrients such as selenium, alpha-tocopherol, and omega-3 fatty acids has been demonstrated, which in particular increased the risk of pancreatic cancer, while carotenoids - the risk of lung cancer (LC), and a complex of biologically active dietary supplements with antioxidants (vitamins C, E, selenium, etc.) cancer of the bladder.

In addition, there is an increased risk of pancreatic cancer with an excessive consumption of fruits and vegetables rich in fructose. The anti-cancer effect of fruits and vegetables depends on the ways they are cooked: fresh fruit and raw vegetables (including frozen ones) are the most useful; vegetables containing carotenoids are more useful when boiled or stewed; while vegetables preserved by means of pickling and marinating, as well as fried vegetables increase the risk of developing certain types of cancer, mainly in digestive organs. To reduce the risk of cancer, it is not so much the use of certain groups of fruits and vegetables that have the most pronounced preventive effect on certain types of cancer, but rather the overall anti-cancer nature of the entire diet, like Mediterranean one and/or the "healthy eating" principles adopted in the main countries of the world. At the same time, it has been proven that nutrition should be anti-cancer, at least from adolescence, or even from early childhood. Given a variety of causes and different mechanisms of development of individual forms and localizations of malignant tumors, a maximally differentiated approach is needed in the evaluation of both individual products and not only individual cancer localizations, but also their varieties within each localization, including histological type of tumors, and also genetic differences. In addition, it is important to conduct research with qualitative design, which involve large cohorts, eliminating systematic or accidental errors, and also taking into account not only favorable dynamics of laboratory indicators, but in the first place, clinically relevant outcomes (mortality and life expectancy of healthy persons, quality of life, metastasis rate, angiogenesis and tumor proliferation in patients, etc.) $[15,16]$.

The results of most studies testify to the need for adequate consumption of vegetables and fruits to prevent the development of cancer in virtually all localizations. No more than sufficient, because their excess can be dangerous, for example, excessive consumption of fruits and berries rich in fructose may increase the risk of pancreatic cancer, of potato - the risk of renal cell carcinoma, as well as a lower risk of certain cancers with an average consumption of vegetables and fruits, compared to their maximum and minimum consumption. So, how much and what vegetables and fruits should be eaten a day to reduce the risk of developing cancer? According to different authors, recommended levels of consumption of vegetables vary from 240 to 402 grams per day, fruits - from 160 to 460 grams per day, dietary fiber - from 25 grams per day. The existing rates of consumption lack specific rates for individual groups of fruits, berries and vegetables.

\section{Conclusion}

Analysis of current information on the effects fruits and vegetables have on the risk of cancer indicates that the problem is understudied. This is confirmed by inconsistency of the data 
provided by different researchers, sometimes arriving at completely opposite conclusions. This is due to the use of different techniques of conducting observations, grouping data, and their evaluation. However, even at the current stage of the development of evidencebased nutritional science, it is possible to identify fairly clear the regularities and repeatedly verified trends that allow drawing certain conclusions and outline the ways for further solving the problem of preventing cancers of various localizations with the help of vegetables and fruits in one's diet.

\section{References}

1. Doll R (1984) The causes of cancer. Quantitative assessment of removable risk factors for cancer in the United States: Per. With the English. In: R Doll, R Pito, Per Yu D Ivashchenko (Eds.). AI Buckwheat - Kiev: Naukova Dumka, Ukraine, p. 256.

2. (2003) Diet, nutrition and the prevention of chronic diseases. Geneva (WHO Technical Series no. 916) p. 156.

3. (2010) World Cancer Research Fund. Washington p. 33.

4. (2011) World Cancer Research Fund. Washington p. 43.

5. (2013) World Cancer Research Fund. Washington p. 37.

6. (2014) World Cancer Research Fund. Washington p. 30.

7. (2014) World Cancer Research Fund International. Washington p. 53.

8. Wang X, Ouyang Y, Liu J, Zhu M, Zhao G, et al. (2014) Fruit and vegetable consumption and mortality from all causes, cardiovascular disease, and cancer: systematic review and dose-response meta-analysis of prospective cohort studies. BMJ p. 14.

9. Smith Warner SA, Genkinger J, Giovannucci E (2006) Fruit and vegetable consumption and cancer. In ( $2^{\text {nd }} E d n$.). Nutritional oncology. San Diego, London, UK, pp. 97-173.

10. Kruk J (2014) Association between Vegetable, Fruit and Carbohydrate Intake and Breast Cancer Risk in Relation to Physical Activity. Asian Pac J Cancer Prev 15(11): 4429-4436.

11. Afrin S, Giampieri F, Gasparrini M, Forbes Hernandez TY, Varela López A, et al. (2016) Chemopreventive therapeutic effects of edible berries: A focus on colon cancer prevention and treatment. Molecules 21(2): 169.

12. Kula M, Krauze Baranowska M, Rubus occidentalis (2016) The black raspberry - its potential in the prevention of cancer. Nutr Cancer 68(1): 18-28.

13. Mentor Marcel RA, Bobe G, Sardo C, Wang L Sh, Kuo Ch T, et al. (2012) Plasma cytokines as potential response indicators to dietary freezedried black raspberries in colorectal cancer patients. Nutr Cancer 64(6): 820-825.

14. Aune D, Chan DS, Lau R, Vieira R, Greenwood DC, et al. (2011) Dietary fibre, whole grains, and risk of colorectal cancer: systematic review and dose-response meta-analysis of prospective studies. Br Med J p. 343.

15. AB Awad, PG Bradford (2006) Nutrition and cancer prevention. by London, p. 618.

16. D Heber, GL Blackburn, VL W Go, J Milner Nutritional oncology (2006) ( $2^{\text {nd }}$ Edn.). San Diego, London, UK, p. 822.
ISSN: 2574-1241

DOI: 10.26717/BJSTR.2018.09.001793

VV Zakrevskii. Biomed J Sci \& Tech Res

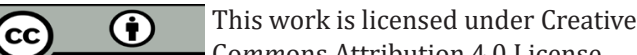

Submission Link: https://biomedres.us/submit-manuscript.php

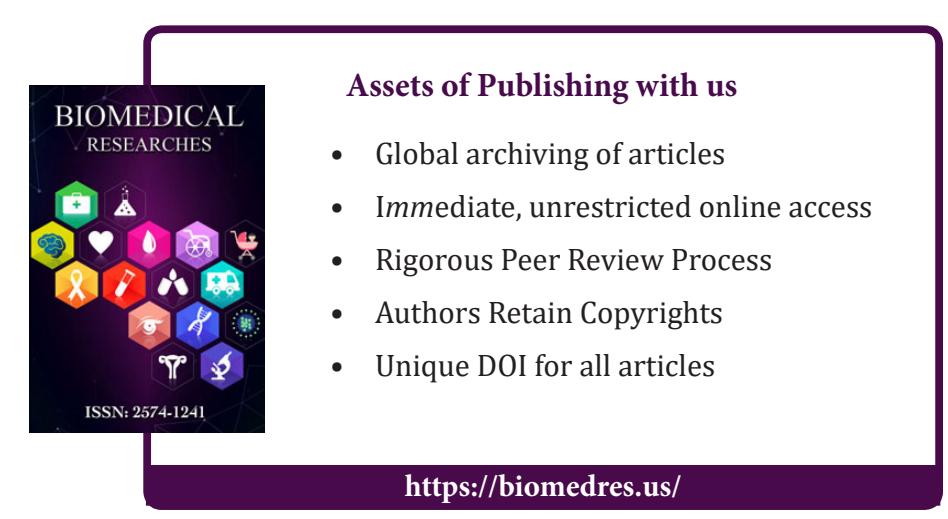

